\title{
e-Science in Scandinavia
}

\author{
The Case of the Swedish e-Science Research Center
}

Olivia Eriksson · Erwin Laure

Erik Lindahl · Dan Henningson

Anders Ynnerman

\section{The Swedish e-Science Research Centre (SeRC)}

The Swedish e-Science Research Centre (SeRC) is based on a collaboration between four Swedish universities: The KTH Royal Institute of Technology (KTH), Stockholm University (SU), Karolinska Institutet (KI) and Linköping University (LiU). SeRC's mission statement is to develop state-of-the-art eScience tools and provide e-infrastructure support to existing and emerging e-Science research communities to help bring about scientific breakthroughs in Sweden. SeRC was founded in 2010 as the result of the Strategic Research Area (SRA) initiative launched by the Swedish Government Bill on Research Policy in 2008 , where a total of 24 different strategic research areas were defined - one of which was e-Science. Initially SeRC was granted funding for 5 years. During those first 5 years, SeRC built up an organization for e-Science research, which has been highly successful. This was reflected in the excellent grades that SeRC received when the SRAs in Sweden were evaluated in 2015, and the fact that after this, SeRC received funding for at least 5 more years. This new phase of SeRC partly focuses on activities relating to emerging technologies (such as exascale systems and data-driven science) while also consolidating SeRC's ongoing efforts in working towards a long-lasting e-Science environment in Sweden.

\section{e-Science and SeRC}

We are right in the middle of a revolution where computers are redefining the process of scientific research as we know it. While this trend started in the natural and engineering sciences, it has evolved to become a pervasive component of virtually all scientific fields: modern aeroplane design depends more on computational fluid dynamics than on wind tunnels, bioinformatics has turned biology into a quantitative subject, and we are seeing entirely new data-driven research, for instance in medicine.

Many of the challenges that are being faced within e-Science are similar across different scientific disciplines;. all these research directions rely on having access to large computational resources and the ability to work with and archive huge amounts of data, and they also need new algorithms (for performing both computations and large-scale analysis of data). Consequently, there is also a demand for e-Science experts, computer experts who are highly skilled in a particular applied research area and also in e-infrastructures or method development.

\section{SeRC's scope, model, and mission}

The concept of e-Science, in its most basic form, is simply the notion of using digital information and processing that information to gain new scientific insights. This means that a lot of e-Science research can be conducted using the standard hardware and software that are widely available. However, there are also research problems that require additional resources, which is where SeRC comes in.

\footnotetext{
https://doi.org/10.1007/s00287-018-01133-2 (c) The Author(s) 2018. This article is available on SpringerLink.com with Open Access.

Olivia Eriksson · Erwin Laure · Erik Lindahl

Dan Henningson - Anders Ynnerman

SeRC, SE-100 44 Stockholm, Sweden

E-Mails: olivia@mech.kth.se, erwinl@pdc.kth.se,

erik.lindahl@scilifelab.se, henning@mech.kth.se,

anders.ynnerman@liu.se
} 
SeRC's scope is to address the e-Science problems that need solutions which go beyond the standard ones that are currently available, and that require researchers to have access to a state-ofthe-art e-infrastructure as well as new e-Science methodology and technology. SeRC is thereby pushing the development of new e-Science tools forward. In this way, SeRC is paving the path for new high-end e-Science that will eventually mature and benefit not only the groups involved in SeRC but also the whole academic base of e-Science users.

To address these kinds of problems, SeRC is based on a three-pillar model (Fig. 1). The three pillars of existing excellence in application areas, core e-Science and method development, and computational infrastructures interact to find new e-Science solutions. In particular, this is done through collaborations and e-Science experts active in at least two of these pillar areas. For the long term, SeRC is working on developing a "human e-cloud" in order to better address challenges that are common to different application areas, and also to provide clear career paths for this new kind of e-Science researchers with dual expertise.

\section{Pillar 1: application areas}

One of SeRC's central goals is to have a direct impact in application areas of strategic relevance for our partner universities, and we also want to focus on research topics where SeRC has world-leading research groups. These SeRC application areas include fields

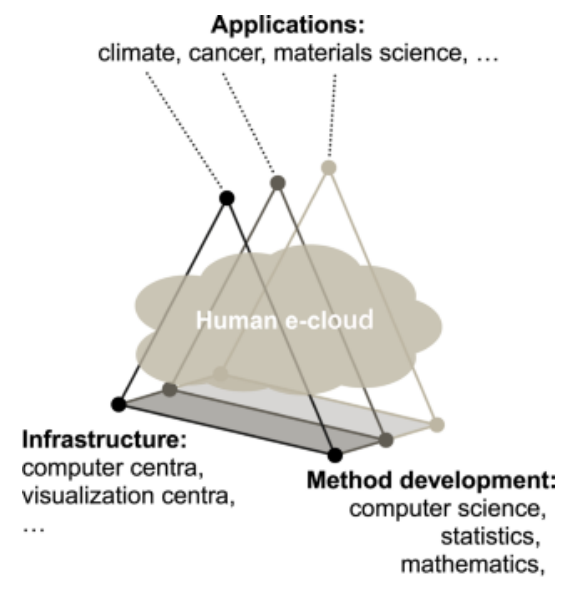

Fig. 1 Three pillars of existing excellence in application areas, method development, and e-infrastructures, connected through a "human e-cloud" such as computational fluid dynamics, materials science, bioinformatics, cancer epidemiology and screening, neuroinformatics, molecular simulation, and climate modeling.

\section{Pillar 2: e-infrastructures}

SeRC wants to facilitate the development of a worldclass computational infrastructure at the PDC Center for High Performance Computing at KTH, the National Supercomputer Center at Linköping University, and at the visualization centers affiliated with SeRC (which are based in Norrköping and at KTH in Stockholm). The development of this infrastructure includes competitive hardware investments in collaboration with the Swedish National Infrastructure for Computing (SNIC), although it is equally important to recruit advanced infrastructure experts to develop key simulation codes further.

\section{Pillar 3: core e-Science and method development}

An integral part of SeRC is leading-edge research in core e-Science and method development, for instance in fields such as numerical analysis, visualization, parallelization, acceleration, and data engineering. SeRC has several core research groups that provide both the latest research results for eScience tools, as well as a broad competence base in e-Science tool usage and methodology.

\section{The human e-cloud}

For SeRC, the central challenge is to get these three pillars to strengthen and support each other through collaborations. Infrastructures and core e-Science do not exist in a vacuum but need to engage directly in research on important applications. In particular, SeRC focuses on supporting application areas where we see an opportunity to translate advances in fundamental e-Science into research impact.

Our primary tool to achieve this is the recruitment of cross-disciplinary e-Science experts that work between these areas - we call them the "human e-cloud" in Fig. 1. These experts combine a deep knowledge of an application area with expertise either in method development or large-scale computational infrastructures. By integrating the application experts into the applied research environments (while they work close to the method development groups or the infrastructure), it 


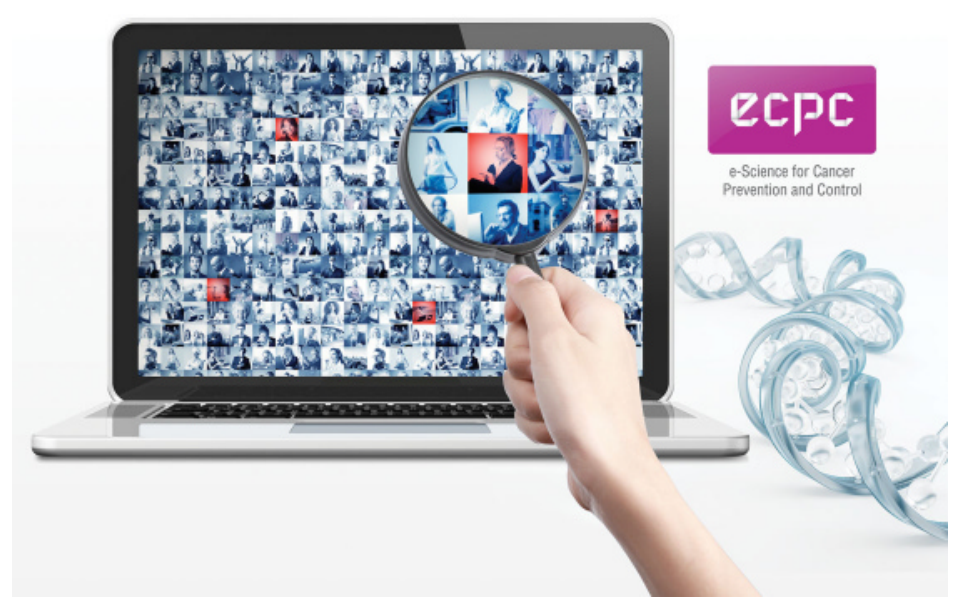

Fig. 2 Example of SeRC collaboration provides opportunities for close collaborations between e-Science experts and applied researchers, as well as between e-Science experts facing similar challenges (for instance, in parallelization, data management, or method development) in different application areas.

Figure 2 introduces "e-Science for Cancer Prevention and Control" as an example of SeRC collaboration. This program has an e-Science team that includes epidemiologists, bioinformaticians, image analysts, and computer scientists who develop, implement, and evaluate methods for using large amounts of biomedical data for risk-stratification in cancer screening and diagnosis. More details are available at http://www.e-science.se/ecpc.

\section{Organization}

SeRC is based on a number of research communities, together with the SeRC e-Science infrastructure. Some of the SeRC research communities focus on core e-Science methodology, like numerical analysis, parallel software and data engineering, and visualization, whereas others focus on applying the methodology in a particular area, like bioinformatics, neuroinformatics, cancer epidemiology and screening, molecular simulation, electronic structure, computational fluid dynamics, or climate research. The infrastructure on the other hand consists of:

- two supercomputer centers - the PDC Center for High Performance Computing at KTH (PDC) and the National Supercomputing Center at LiU (NSC), both of which are part of SNIC.
- Two visualization centers - the Visualization Center C (VC), located in Norrköping, and the Visualisation Studio at KTH (VIC).

- A number of e-Science experts who are employed at the supercomputer center, and who work closely with the research communities.

SeRC has been co-financing about 30 projects and 15 faculty positions per year but is now in the process of reallocating the funding to a number of larger multidisciplinary collaboration programs initiated in order to increase the level of collaboration between researchers in the areas of applications, methodology, and infrastructure.

To consolidate the organization, in 2013 SeRC was turned into a research center with $\mathrm{KTH}$ as the host, and shortly after we recruited an external chair of the board, Professor Morten Dæhlen, who is Dean of Mathematics and Natural Sciences at the University of Oslo and a prominent international e-Science leader. More information about the SeRC organization can be found at http://www.e-science.se/org.

\section{SeRC faculty}

Since the inception of SeRC, we have recruited a substantial number of highly skilled assistant professors. In addition, we have selected a number of e-Science investigators who were either hired recently or who recently joined SeRC with support from other funding. Together, these researchers form the SeRC faculty.

The main goal of the SeRC faculty is to facilitate interdisciplinary exchange between e-Science 
communities in application areas and core e-Science and method development, and to provide SeRC with a network of e-Science researchers that straddle the various research communities and multidisciplinary collaboration programs.

\section{Collaborations and scientific output}

SeRC hosts a large number of outstanding scientists who are pushing the state-of-the-art, in application areas as well as method development, with several high-impact results. However, SeRC's mission is to go a step beyond merely providing some marginal funding and instead build a scientific environment that promotes excellence within research disciplines through multidisciplinary collaboration. SeRC makes it possible to initiate new, ambitious and risky collaborative projects that have the potential to redefine research fields. In particular, the center has acted as a catalyst in the formation of several strong research environments that work on the application, method development, and infrastructure aspects of computational research (for example, see Fig. 2). These multidisciplinary collaborative projects, for instance, make it possible to engage computational experts in long-term development efforts that have previously been very difficult to fund.

The new initiatives have had tremendous scientific impact: teams that did not even know each other prior to SeRC have merged, and the collaborations between infrastructures and researchers have led to a range of new high-profile international research projects being funded, not to mention the award of several prestigious European Research Council grants. However, instead of being content with these achievements, SeRC will keep raising the bar by continuously evaluating our collaborations with a view to further improving the center's effectiveness. SeRC's goal is not to maintain the status quo, but to promote discoveries and new applications by helping and encouraging talented researchers to team up and leave their comfort zone. Collaboration, multidisciplinarity, change, and impact are the genes of SeRC.

\section{Computing resources}

e-Science is critically dependent on efficient largescale computing and storage resources. Within SeRC, these resources are provided by the leading Swedish high performance computing centers, PDC and NSC, which offer more than two-thirds of the
Swedish national computing capacity. A strategic partnership between these centers has been established. This allows for better coordination and alignment of their services with the needs of SeRC researchers.

Since 2015, SeRC researchers have had access to petascale computing resources through these centers. However, it is noteworthy that PDC and NSC not only provide hardware, they also provide expertise in many application areas in terms of computing experts who work closely with certain scientific domains, including bioinformatics, climate research, computational fluid dynamics, materials sciences, molecular dynamics, and neuroinformatics.

In January 2015, the SeRC infrastructure received a significant boost with the inauguration of Beskow at PDC - a 2-petaflop Cray XC40 system that is more powerful than all the other SNIC systems together. With Beskow, SeRC researchers had a world-class petascale system at their disposal for the first time - it was much needed for their research and also as a stepping stone towards using the larger European-level HPC infrastructure. Beskow was designed to cater for the needs of SeRC and important SeRC applications were used as benchmarks to guide the selection process.

In the autumn of 2017, NSC's flagship system, Triolith, was replaced with another petascale system, ensuring that SeRC researchers and other e-Science researchers in Sweden continue to have access to the latest hardware technologies.

\section{The future and e-Science agenda}

During its first 5 years, SeRC had tremendous success in establishing e-Science in Sweden and fostering the e-Science paradigm in many research areas. e-Science is now well established and is a priority area at the SeRC partner universities. Starting up the next phase of SeRC we are consolidating these efforts by working towards a long-lasting e-Science environment and, on the other hand, increasing SeRC activities relating to emerging technologies for e. g. exascale systems and data-driven science.

The report Swedish Science Cases for e-Infrastructure (http://www.e-science.se/sscei) is an important document when it comes to shaping the future of e-Science in Sweden. This document discusses strategic e-Science development in Sweden and makes a number of recommendations that 
are of help in guiding future SeRC developments. Key findings, from a SeRC perspective, include the following.

- "Development of methods, tools and software within core disciplines is necessary to make breakthroughs."

- "Advanced and long-term user support and human infrastructures are keys to e-Science adoption."

- "The simulation paradigm dominates the current Swedish needs for e-infrastructure. A complementary and more data-centric aspect of e-Science should be promoted."

- "e-Social science and e-humanities are potentially very large users, but need active support, like other research communities new to e-Science."

- "e-Science methods and tools are in increasing demand and will be instrumental in increasing interaction between tool makers and tool users."

Responding to these findings entails work at several levels, ranging from infrastructure and tool development to policy definitions. In its strategy process, SeRC has defined an agenda for the second phase. This agenda addresses many of the items from the report and describes how SeRC is in a unique position to spearhead this work.

\section{The impact of e-Science}

The impact of e-Science can be seen in all areas of academia. The use of basic e-Science in new research domains can generate fundamentally new and important results. At the same time, access to advanced e-Science tools and infrastructures enables the leading research groups that are spearheading Swedish e-Science to compete at the international forefront. In view of this, SeRC intends to intensify the collaboration with relevant Swedish groups and universities that are currently not part of SeRC, in domains like bioinformatics, numerical analysis, and materials science. Also, the broader adoption of e-Science methodology cannot be overestimated. Through extensive collaborations with industry and governmental agencies, SeRC has a direct impact in society and contributes to societal development and commercial competitiveness. SeRC has several industrial representatives on its advisory board, and we are delighted with their interest and engagement in learning how computing can change what they do, for instance at our annual meetings. One of the primary indirect mechanisms for SeRC to have an effect is through knowledge transfer in the form of recent Ph.D. graduates, who have unique experiences of e-Science and form a network consisting of the next generation of e-scientists.

The single largest overall success of SeRC is that it has helped application researchers and computational experts to find each other and speak with one voice. Large-scale data analysis and simulation have been introduced in areas where they have never been used before, the strongest computational groups in the country suddenly collaborate internationally instead of competing nationally, and we will keep redefining these boundaries by identifying areas where the combination of computational and application research excellence makes a field ripe for disruptive changes to the science.

\section{SeRC initiative: multidisciplinary collaboration programs}

In the first phase of SeRC, larger, multidisciplinary constellations were prototyped through a multidisciplinary collaboration program (MCP) promoting multidisciplinary approaches to strategic research areas with high potential for gains. The e-Science for Cancer Prevention and Control (eCPC) was SeRC's first MCP, which was followed by "Visualisation in e-Science Applications" and "SESSI-SeRC Exascale Simulation Software Initiative".

The illustration from the SeRC Multidisciplinary Program "Visualisation in e-Science Applications" (Fig. 3) shows a visualization of a protein, combining hybrid data from different sources using a hybrid visualization of geometric protein representations and volumetric data. The improved A-buffer algorithm is capable of rendering the entire scene with a performance increase of 5.9 times compared to prevalent techniques.

The illustration from the SeSSI MCP (Fig. 4) shows results from computational modeling of the skin barrier (the lipid matrix of the stratum corneum) using molecular dynamics simulations, which gives us further insight into the largest organ of the human body and will also further clinical experiments. Thanks to the highly optimized heterogeneous parallelization in the GROMACS code, complex computational studies can be carried out quickly and efficiently. The image on the right shows the modeled molecular system with ceramide 


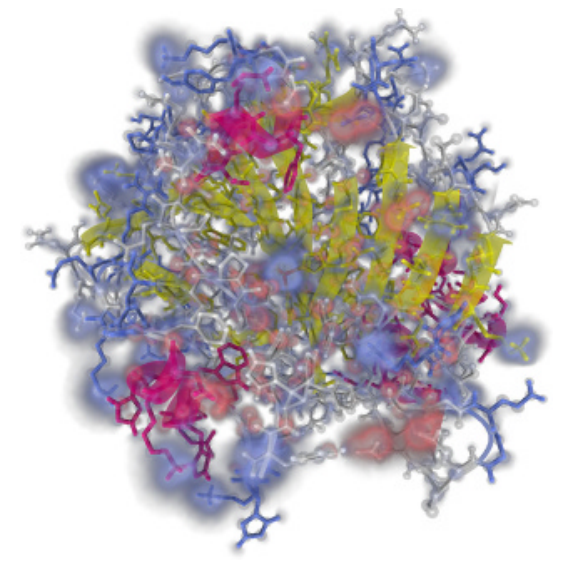

Fig. 3 Illustration from the SeRC multidisciplinary program "Visualisation in e-Science Applications"

molecules in green, cholesterols in white, and fatty acids in red. Recent work in SeSSI (that focused on improving SIMD, GPU, and thread parallelization) resulted in speeding up the calculations by up to $50 \%$. The figures on the left show the execution time breakdown of the CPU and GPU tasks in GROMACS versions 5.1 and 2016; improved performance of multiple tasks leads to an increase in simulation throughput from $61 \mathrm{~ns} /$ day to $95 \mathrm{~ns} /$ day. The simulations were performed on a workstation equipped with a Core i7-5960X CPU and a GeForce TITAN X GPU.

SeRC will now build on these successes and further develop the MCPs by consolidating the SeRC efforts into larger constellations. A key requirement of MCPs is the existence of external third-party support contributing to the program goals. These multiple funding streams are one component to ensure the sustainability of SeRC efforts. Recently, three additional MCPs have been started: Data-Driven Computational Materials Design, SeRC Data Science, and Brain-IT.

\section{SeRC initiative: scientific computing lab}

SeRC groups are engaged in the development of computational methods and infrastructures, which are necessary ingredients for world-class computer-based scientific research. With architectural changes, which are being driven partly by the challenge of building exascale systems, these efforts need to be reinforced and strengthened. Developments - such as massively increased core counts, large vector units, deep memory hierarchies, and the like - require significant efforts in the development of efficient, scalable methods and implementations.

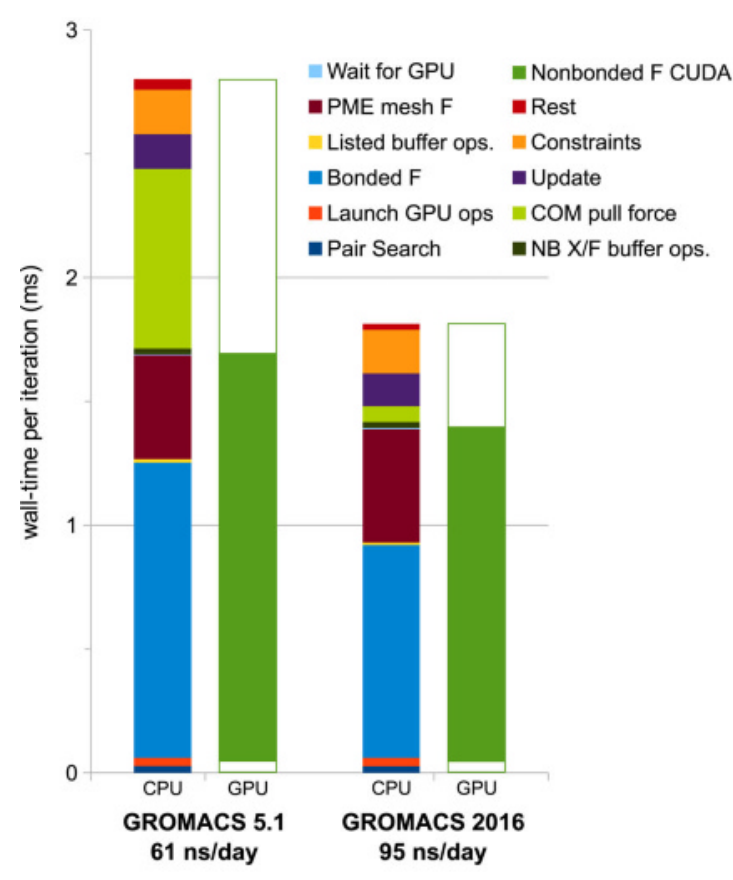

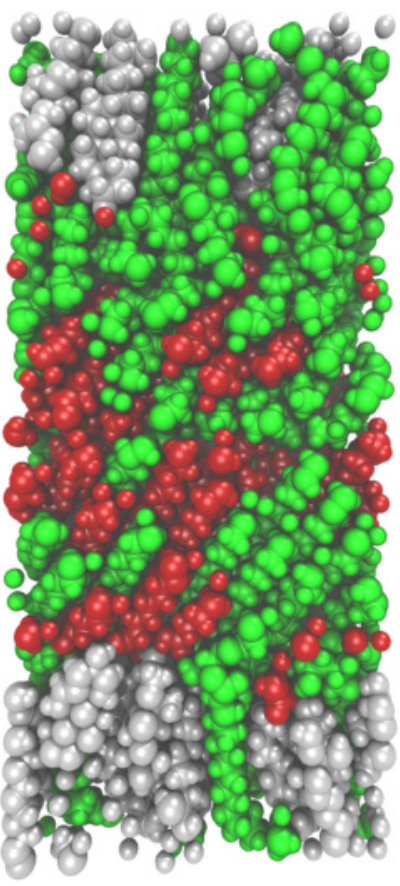

Fig. 4 Illustration from the SeSSI MCP. (Source: https:// doi.org/10.6084/m9.figshare. 3423395.v1 (Magnus Lundborg and Szilárd Páll) image reused under (C-BY) 
Such work is planned in the MCPs referred to earlier. These constitute ideal cases for the SeRC vision of integrating researchers (from the three pillars that were described earlier) with each other in such a way that collaboration between application area, e-infrastructure, and methods researchers is being facilitated by the e-Science experts in the human e-cloud.

Following international examples, for instance from the National Center for Supercomputing Applications at the University of Illinois at UrbanaChampaign, we intend to prototype a scientific computing lab, where researchers working on applications, methods, and infrastructure can effectively collaborate in such a manner. A physical interaction space that has been created at PDC is a key component in this endeavor. With this lab, SeRC will build the nucleus of the human e-cloud identified as a key ingredient above.

\section{SeRC initiative: data-driven science}

The data deluge that is arising from new scientific instruments, large simulations, and data on the internet, together with new technologies for handling and analyzing this data, has significant impact on eScience methodology. SeRC is, therefore, embracing big data, with an initial emphasis on the life sciences. This includes research regarding storage, manage- ment, integration, visualization, and analysis of big data.

In recent years, new platforms and tools have appeared that can efficiently and cost-effectively store and process up to petabytes of data. This allows many organizations to handle data that was previously considered to be too expensive to store and manage. This data-driven science trend is transforming scientific research, by making data-driven discovery and prediction possible. The requirements for data analytics on a large scale include data management on public and private clouds, dataparallel algorithms, and proficiency with a complex ecosystem of tools and platforms such as Hadoop. Currently, these are not widely available within the existing SeRC research communities, so one of the goals for SeRC is to help transfer knowledge about the use of methods and tools from data science to facilitate the use of new research methodologies in other SeRC communities.

Open Access. This article is distributed under the terms of the Creative Commons Attribution Noncommercial License (http://creativecommons.org/ licenses/by/4.o/deed.de) which permits any noncommercial use, distribution, and reproduction in any medium, provided the original author(s) and source are credited. 